Note added in proof: Only in one of 137 cases of decay is a $\gamma$-quantum emitted. In all other cases an unobservable and monochromatic neutrino radiation appears. Prof. W. Pauli remarked in a discus. sion that this radiation must be followed by emission of the characteristic X-ray spectrum of the atom preceding the unstable element in the periodic system. A complete account will be published in the Helv. Phys. Acta.

Institute of Physics,

$$
\text { E. C. G. STuECKelberg. }
$$

University of Geneva. May 7.

1 Bloch and Moller, Nature, 186, 911 (1935); M. Fierz, Helv. Phys. Acta, 9, 245 (1936); G. Rumer, Sow. Phys., 9, 317 (1936).

\section{Distribution of Atomic Nuclear Spins}

I HAVE previously pointed out ${ }^{1}$ that the distribution of atomic nuclear spins appears to be entirely different in the two types of odd atomic weight nuclei, namely, class $A$, those possessing an odd nuclear proton, and class $B$, those possessing an odd nuclear neutron. Since then quite a number of nuclear spins have been added to those known, data now being available for sixty-four elements, among which are seventy odd

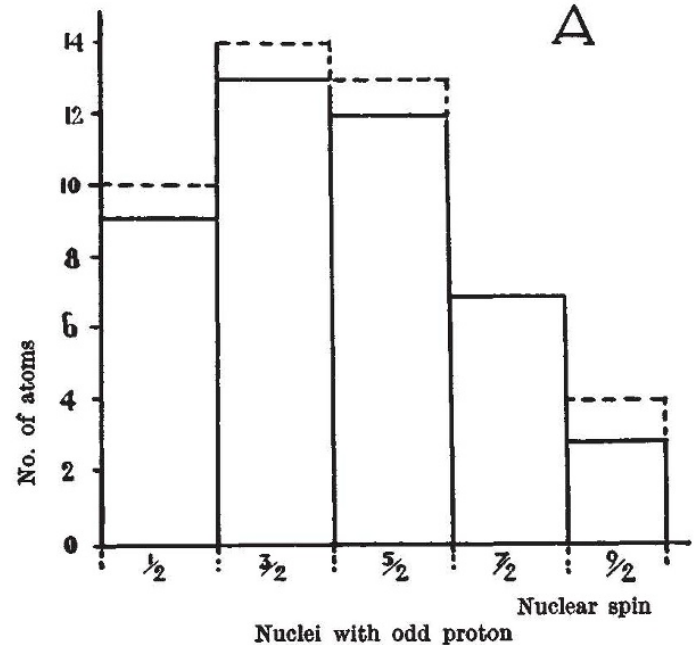

to arise from orbital moments ( $l$ values) of 0,1 and 2 (and possibly even from 3 and 4 if cases exist in which five neutron spins add together). Further, $l$ values of 2 and 3 would tend to give nuclear spins of $\frac{3}{2}$ and so on. This view, therefore, accounts for the preponderance of spins of $\frac{1}{2}$ and of low values in general. In class $A$, only $l$ values of zero produce spins of $\frac{1}{2}$ with certainty, $l$ values of 1 resulting in both $\frac{1}{2}$ and $\frac{3}{2}$ spin values.

The distribution of the spins of even atomic weight atoms has received practically no attention. In $1932^{4}$ I pointed out a relationship between nuclear spin and mass defect in the lighter elements. Only the atoms on the lower spur of the mass defect curve (atomic weights multiples of 4) definitely have zero nuclear spin. All the odd atoms and a number of even atoms lie on the upper spur. It was suggested that the less tightly bound particles which cause the large positive mass defect are likely also to produce nuclear spin. At that time the spin of ${ }^{14} \mathrm{~N}=1$ was the only spin greater than zero known to be exhibited by an even atom. I predicted, from the mass defect curve, spins of 1 for ${ }^{6} \mathrm{Li}$ and ${ }^{10} \mathrm{~B}$. We now know that the spins of the atoms ${ }^{2} \mathrm{H},{ }^{6} \mathrm{Li},{ }^{14} \mathrm{~N}$ are equal to 1 . All lie on the upper spur, and therefore it is now very reasonable to predict a spin of 1 for ${ }^{10} \mathrm{~B}$.

FIG. 1. Distribution of nuclear spins among the odd atomic weight atoms.

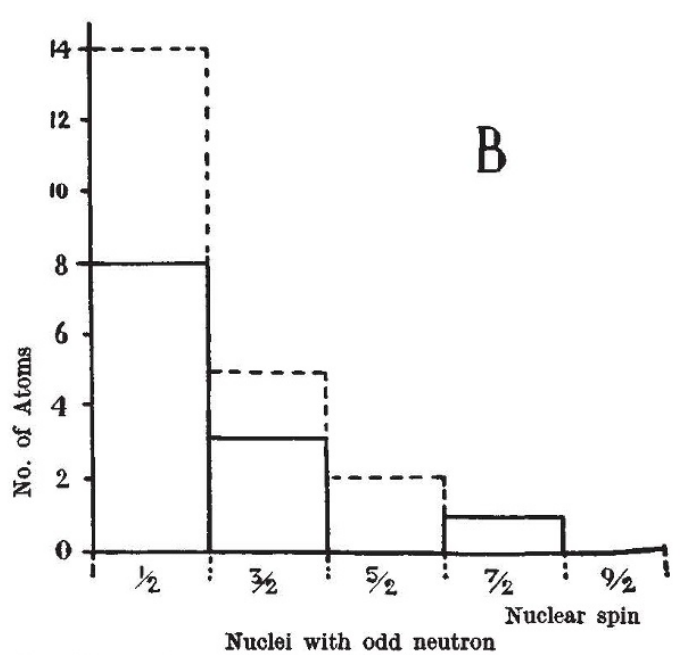

isotopes and seven even atoms. Improved distribution curves can thus be drawn, as in the accompanying diagram (Fig. 1). The full lines show the spin values known with certainty, and above these (dotted) are included the spins about the value of which there is yet some doubt (generally occasioned by the observation of fine structure in terms with $J$ values insufficient to permit full fine structure multiplicity to be reached).

Inglis ${ }^{2}$ has discussed this distribution with relation to the stabilities of the nuclear particles. The most striking feature of the curves is the preponderance of the spin $\frac{1}{2}$ in class $B$ nuclei (and of low values in general in this group). The improved curves show even better than before, that, as I have already suggested, in class $B$, neutron spin and orbital moments tend to oppose each other. This explains the differences in the two curves. According to the theory of Tamm and Altschuler ${ }^{3}$ the total neutron spin in a nucleus can have the value $\frac{1}{2}$ or $\frac{8}{2}$ according to circumstances. Thus a nuclear spin of $\frac{1}{2}$ will tend
With regard to ${ }^{18} \mathrm{O}$ and onwards, Bartlett's evidence $e^{5}$ for the beginning of a new nuclear shell at ${ }^{16} \mathrm{O}$ suggests that any prediction for ${ }^{18} \mathrm{O}$ and onwards must be accepted with caution. Thus in Bartlett's first group, the even atoms divisible by four all have zero spin and the others have a spin of 1 , if the view put forward here is correct. In the latter cases the spin is due to the odd neutron and proton combining with mechanical moments in parallel, and as the magnetic moments of these particles are of opposite signs, the resulting $g(I)$ factors will be small and therefore the fine structures will be minute. This has been observed in the fine structures of ${ }^{6} \mathrm{Li}$ and ${ }^{14} \mathrm{~N}$, and may be expected for ${ }^{10} \mathrm{~B}$.

Physics Department,

S. Tolansky.

\section{University, Manchester.} April 22.

1 NATURE, 135, 620 (1935)

Inglis, NATURE, 135, 998 (1935).

3 Tamm and Altschuler, C.R. Acad. Sci. U.S.S.R., 1, 455 (1934)

- Z. Phys., 78, 71 (1932)

S NATURE, 130, 165 (1932). 